\title{
Dinkelbach Approach to Solving a Class of Fractional Optimal Control Problems
}

\author{
I. Bykadorov • A. Ellero • S. Funari · E. Moretti
}

Published online: 5 March 2009

(C) Springer Science+Business Media, LLC 2009

\begin{abstract}
We consider optimal control problems with functional given by the ratio of two integrals (fractional optimal control problems). In particular, we focus on a special case with affine integrands and linear dynamics with respect to state and control. Since the standard optimal control theory cannot be used directly to solve a problem of this kind, we apply Dinkelbach's approach to linearize it. Indeed, the fractional optimal control problem can be transformed into an equivalent monoparametric family $\left\{\mathrm{P}_{\mathrm{q}}\right\}$ of linear optimal control problems. The special structure of the class of problems considered allows solving the fractional problem either explicitly or requiring straightforward classical numerical techniques to solve a single equation. An application to advertising efficiency maximization is presented.
\end{abstract}

Keywords Optimal control · Fractional programming · Dinkelbach algorithm • Advertising efficiency

Communicated by P.M. Pardalos.

This work was partially supported by the Università Ca' Foscari, Venezia, Italy, the MIUR (PRIN cofinancing 2005), the Council for Grants (under RF President) and State Aid to Fundamental Science Schools (Grant NSh-4113.2008.6).

We thank Angelo Miele, Panos Pardalos and the anonymous referees for comments and suggestions.

I. Bykadorov

Sobolev Institute of Mathematics, Siberian Branch Russian Academy of Sciences, Novosibirsk, Russia

e-mail: bykad@math.nsc.ru

A. Ellero $(\varangle) \cdot$ S. Funari · E. Moretti

Dipartimento di Matematica Applicata, Università Ca’ Foscari, Venice, Italy

e-mail: ellero@unive.it

S. Funari

e-mail: funari@unive.it

E. Moretti

e-mail: emoretti@unive.it 


\section{Introduction}

In this paper, we consider the class of fractional optimal control problems where the functional is given by the ratio of two integrals; this kind of problems has already been tackled by Stancu-Minasian [1], Bhatt [2] and, in a more general framework, by Miele [3]. We focus, in particular, on problems with affine integrands and linear dynamics with respect to state and control.

Despite the simplicity of these problems, the standard optimal control theory cannot directly be used to solve them. Stancu-Minasian [1] suggested to face the general fractional optimal control problem applying the Dinkelbach's method [4, 5], which is used in fractional programming to remove the denominator in the objective function. With Dinkelbach's approach, the fractional optimal control problem is transformed into an equivalent monoparametric family $\left\{\mathrm{P}_{\mathrm{q}}\right\}$ of optimal control problems, where the ratio disappears and the functional is given by the weighted difference of the numerator and the denominator of the ratio.

In the special class of problems with affine integrands and linear dynamics, the family $\left\{\mathrm{P}_{\mathrm{q}}\right\}$ consists of linear optimal control problems. The special structure of the problems that we consider, allows us to solve them either explicitly or requiring classical numerical techniques to solve a single equation.

The paper is organized as follows. In Sect. 2, we formulate the fractional optimal control problem and recall Dinkelbach's linearization approach for fractional programming. In Sect. 3, we face a class of fractional optimal control problems with affine integrands and linear dynamics and find the structure of the optimal control of the corresponding linear optimal control problems.

In Sect. 4, we present the solution of the fractional problem, while in Sect. 5 we discuss an application to advertising campaigns efficiency maximization. Some final remarks and hints for future research are given in Sect. 6.

\section{Fractional Optimal Control Problems}

Let us consider a fractional optimal control problem (see [1]):

$$
\begin{aligned}
(\mathrm{FP}) \max & \frac{\int_{0}^{T} f(x(t), u(t), t) d t}{\int_{0}^{T} g(x(t), u(t), t) d t}, \\
\text { s.t. } & \dot{x}(t)=h(x(t), u(t), t), \\
& x\left(t_{0}\right)=x_{0},
\end{aligned}
$$

where $x(t) \in R^{n}, u(t) \in U \subset R^{m}$ and $t \in[0, T]$. As usual in fractional programming, Stancu-Minasian assumes that the denominator of the fraction is strictly positive, i.e.

$$
\int_{0}^{T} g(x(t), u(t), t) d t>0
$$

for all $u(t) \in U$. 
Stancu-Minasian proposes a numeric procedure to solve (FP). The key idea is to use Dinkelbach's algorithm [4, 5] for fractional programming as modified by Bhatt [2], and by Stancu-Minasian himself, also to solve fractional optimal control problems. The approach consists in transforming the original fractional problem into an equivalent family of parametric optimal control problems, which become non fractional.

More precisely, let us define an auxiliary function $F$ such that, for each $q \in \mathbb{R},{ }^{1}$ $F(q)$ is the maximum value of the following (nonfractional) optimal control problem:

$$
\left(\mathrm{P}_{\mathrm{q}}\right) \max _{(x, u) \in \Omega}\left[\int_{0}^{T}\{f(x(t), u(t), t)-q g(x(t), u(t), t)\} d t\right],
$$

where $\Omega$ is defined by the dynamic system

$$
\dot{x}(t)=h(x(t), u(t), t),
$$

with $x(0)=x_{0}$ and $u \in U$.

If for each $q \in \mathbb{R}$ there exists an optimal control function $u(t) \in U$ for problem $\left(\mathrm{P}_{\mathrm{q}}\right)$, then the function $F$ is strictly decreasing and convex and has a (unique) zero $q^{*}$ (see [1]).

The nice property that relates the original fractional optimal control problem (FP) with the family of auxiliary problems $\left(\mathrm{P}_{\mathrm{q}}\right)$, is that, if $F\left(q^{*}\right)=0$, then $q^{*}$ is the optimal value of (FP) and the optimal control and the optimal trajectory of $\mathrm{P}_{\mathrm{q}}$ are optimal also for problem (FP) (see [1], Theorem 4.6.1, p. 157). It follows that solving problem (FP) is equivalent to determining the root of the equation $F(q)=0$.

Hence, following Dinkelbach's idea, the solution of the fractional problem (FP) can be obtained by means of a Newton-like iterative procedure which starts from a value of $q$ such that $F(q)>0$ determining a sequence of values of $F(q)$ that converges to zero. The effectiveness of this method depends of course on the features of the family of auxiliary optimal control problems.

\section{A Class of Fractional Optimal Control Problems}

Consider the following class of single state and single control fractional optimal control problems:

$$
\begin{array}{ll}
\max & \frac{x(T)+k \int_{0}^{T}(x(t)+c) d t}{u_{0}+\int_{0}^{T} u(t) d t}, \\
\text { s.t. } & \dot{x}(t)=a x(t)+b u(t), \\
& x(0)=x_{0}, \\
& u \in[0, \bar{u}],
\end{array}
$$

where $x(t), u(t) \in \mathbb{R}, a \neq 0, b \neq 0, \bar{u}>0, u_{0}>0$ and $c, k \in \mathbb{R}$.

${ }^{1} \mathbb{R}$ : the set of real numbers. 
Problem $(\mathrm{P})$ has one state variable $x(t)$, continuous and piecewise continuously differentiable, and one control variable $u(t)$, piecewise continuous. Even if $(\mathrm{P})$ is a fractional optimal control problem with a finite horizon, affine integrands and linear dynamics, to solve it we cannot directly use the standard optimal control theory [6-8].

A possible way to solve problem $(\mathrm{P})$ is to use the Dinkelbach's approach as outlined in the previous section. We consider therefore the auxiliary function $F$, defining $F(q)$ as the maximum value of the following optimal control problem, which turns out to be linear:

$$
\left(\mathrm{P}_{\mathrm{q}}\right) \max _{(x, u) \in \Omega}\left[\left(x(T)+k \int_{0}^{T}(x(t)+c) d t\right)-q\left(u_{0}+\int_{0}^{T} u(t) d t\right)\right],
$$

where $\Omega$ is defined by the constraints of Problem $(\mathrm{P})$,

$$
\begin{aligned}
& \dot{x}(t)=a x(t)+b u(t), \\
& x(0)=x_{0}, \\
& u \in[0, \bar{u}] .
\end{aligned}
$$

Remark that, for each fixed $q,\left(\mathrm{P}_{\mathrm{q}}\right)$ can be solved by classical linear optimal control techniques.

Since for each $q \in \mathbb{R}$ there exists an optimal control function $u(t) \in U$ for problem $\left(\mathrm{P}_{\mathrm{q}}\right)$, then as remarked in Sect. 2, the function $F$ is strictly decreasing, convex and has a unique zero $q^{*}$ which is also the optimal value of problem $(\mathrm{P})$.

\subsection{Optimal Control of the Linearized Problem $\left(\mathrm{P}_{\mathrm{q}}\right)$}

The optimal control of problem $\left(\mathrm{P}_{\mathrm{q}}\right)$ is described in Proposition 3.1; to prove it, we need the following lemma.

Lemma 3.1 Let $q=-k b / a$ and $a=-k$. Then, $F(q)=x_{0}+k c T-b u_{0}$.

Proof Using the motion equation of problem $\left(\mathrm{P}_{\mathrm{q}}\right)$, we can rewrite its objective function this way (remark that now $q=b$ ):

$$
\begin{aligned}
F(q) & =\left(x(T)+k \int_{0}^{T}(x(t)+c) d t\right)-q\left(u_{0}+\int_{0}^{T} u(t) d t\right) \\
& =\int_{0}^{T}[k x(t)-q u(t)+k c] d t+x(T)-q u_{0} \\
& =\int_{0}^{T}[k x(t)-q u(t)] d t+\int_{0}^{T} \dot{x}(t) d t+x_{0}+k c T-q u_{0} \\
& =\int_{0}^{T}[k x(t)-q u(t)+a x(t)+b u(t)] d t+x_{0}+k c T-q u_{0} \\
& =x_{0}+k c T-b u_{0} .
\end{aligned}
$$


Let us define the following metaparameter:

$$
L=\left(1+\frac{k}{a}\right) e^{a T}-\frac{k}{a} .
$$

Proposition 3.1 The optimal control $u(t)$ of problem $\left(\mathrm{P}_{\mathrm{q}}\right)$ is the following:

(a) if $b(k+a)<0$ :

$$
\begin{aligned}
& \text { if } q \leq b L, \quad \text { then } u(t)=\bar{u}, \forall t \in(0, T) \text {; } \\
& \text { if } q \in(b L, b), \quad \text { then } u(t)= \begin{cases}0, & \text { if } t \in(0, \tau) ; \\
\bar{u}, & \text { if } t \in(\tau, T) ;\end{cases} \\
& \text { if } q \geq b, \quad \text { then } u(t)=0, \forall t \in(0, T) \text {; }
\end{aligned}
$$

where the switching time $\tau$ is given by

$$
\tau=T-\frac{1}{a} \log \frac{k b+a q}{b(k+a)} .
$$

(b) If $b(k+a)>0$ :

$$
\begin{aligned}
& \text { if } q \leq b, \quad \text { then } u(t)=\bar{u}, \forall t \in(0, T) \text {; } \\
& \text { if } q \in(b, b L), \quad \text { then } u(t)= \begin{cases}\bar{u}, & \text { if } t \in(0, \tau) ; \\
0, & \text { if } t \in(\tau, T) ;\end{cases} \\
& \text { if } q \geq b L, \quad \text { then } u(t)=0, \forall t \in(0, T) \text {. }
\end{aligned}
$$

(c) If $b(k+a)=0$ :

$$
\begin{aligned}
& \text { if } q<b, \quad \text { then } u(t)=\bar{u}, \forall t \in(0, T) \\
& \text { if } q>b, \quad \text { then } u(t)=0, \forall t \in(0, T) \\
& \text { if } q=b, \quad \text { then any feasible control is optimal. }
\end{aligned}
$$

Proof Given problem $\left(\mathrm{P}_{\mathrm{q}}\right)$ consider its Hamiltonian function

$$
H_{q}=k x(t)-q u(t)+\lambda(t)[a x(t)+b u(t)]=[k+\lambda(t) a] x(t)+[\lambda(t) b-q] u(t),
$$

where, due to the Pontryagin maximum principle, the function $\lambda(t)$ satisfies

$$
\begin{aligned}
& \dot{\lambda}(t)=-a \lambda(t)-k, \\
& \lambda(T)=1,
\end{aligned}
$$

i.e.,

$$
\lambda(t)=\left(1+\frac{k}{a}\right) e^{a(T-t)}-\frac{k}{a} .
$$


Therefore, the switching function is

$$
G_{q}(t)=\lambda(t) b-q=\frac{b(a+k)}{a} e^{a(T-t)}-\frac{b k}{a}-q .
$$

Observe that, given (1), $G_{q}(0)=L b-q$ and $G_{q}(T)=b-q$.

(a) Let be $b(a+k)<0$. The function $G_{q}(t)$ is strictly increasing. Therefore we have that if $G_{q}(0) \geq 0$, i.e. $L b-q \geq 0$, then the optimal control is $u(t)=\bar{u}$ and if $G_{q}(T) \leq 0$, i.e. $b-q \leq 0$, then the optimal control is $u(t)=0$.

If instead $G_{q}(0)<0$ and $G_{q}(T)>0$, we can determine the (unique) time $\tau \in$ $(0, T)$ to switch from $u(t)=0$ to $u(t)=\bar{u}$ solving the equation $G_{q}(t)=0$. The switching time is therefore

$$
\tau=T-\frac{1}{a} \log \frac{k b+a q}{b(k+a)} .
$$

Observe that in this case $(k b+a q) /(b(k+a))>0$ and then $\tau$ is well defined.

(b) If $b(a+k)>0$ function $G_{q}(t)$ is strictly decreasing and the optimal control is $u(t)=\bar{u}$ if $G_{q}(T) \geq 0$ while it is $u(t)=0$ if $G_{q}(0) \leq 0$. If $G_{q}(0)>0$ and $G_{q}(T)<0$ we have to switch from $u(t)=\bar{u}$ to $u(t)=0$ at time $\tau$. Also in this case $(k b+a q) /$ $(b(k+a))>0$, and $\tau$ is well defined.

(c) In case $b(a+k)=0$, i.e. $a=-k$ since $b \neq 0$, function $G_{q}(t)$ is constant and its sign coincides with the sign of $-(b k+a q) / a$, i.e. with the sign of $b-q$ : therefore the optimal control turns out to be $u(t)=\bar{u}$ if $q<b$ and $u(t)=0$ if $q>b$. In the special case $b k+a q=0$, i.e. $q=b$, then the switching function is identically zero. In this case it is not possible to apply the Pontryagin Maximum Principle but, due to Lemma 3.1, function $F(q)$ turns out to be constant in this case and any feasible control is optimal for $\left(\mathrm{P}_{\mathrm{q}}\right)$.

\section{Solution of Problem (P)}

\subsection{Analytical Expression of the Function $F(q)$}

In order to obtain an explicit solution of problem (FP), let us compute the expression of the function $F(q)$ in analytical form. Recalling (1) we obtain the following proposition.

Proposition 4.1 The function $F(q)$ is the following:

(a) if $b(k+a)<0$, then

$$
F(q)= \begin{cases}F_{1}(q), & \text { if } q \leq b L \\ F_{2}(q), & \text { if } b L<q<b \\ F_{3}(q), & \text { if } q \geq b\end{cases}
$$


(b) if $b(k+a)>0$, then

$$
F(q)= \begin{cases}F_{1}(q), & \text { if } q \leq b \\ F_{4}(q), & \text { if } b<q<b L \\ F_{3}(q), & \text { if } q \geq b L\end{cases}
$$

(c) if $b(k+a)=0$, then

$$
F(q)= \begin{cases}F_{1}(q), & \text { if } q \leq b \\ F_{3}(q), & \text { if } q \geq b\end{cases}
$$

where

$$
\begin{aligned}
F_{1}(q)= & -\left(u_{0}+\bar{u} T\right) q+x_{0} L+c k T-\frac{b \bar{u}}{a}(1-L+k T), \\
F_{2}(q)= & -\left(u_{0}-\frac{\bar{u}}{a}\right) q+x_{0} L+c k T-\frac{b \bar{u}}{a}\left[1+\frac{a q+k b}{a b} \log \frac{a q+k b}{b(a+k)}\right], \\
F_{3}(q)= & -u_{0} q+x_{0} L+c k T \\
F_{4}(q)= & -\left(u_{0}+\frac{\bar{u}}{a}\right) q+x_{0} L+c k T \\
& +\frac{b \bar{u}}{a}\left\{L+\frac{a q+k b}{a b}\left[-a T+\log \frac{a q+k b}{b(a+k)}\right]\right\}
\end{aligned}
$$

Proof Substituting in the motion equation the optimal control $u(t)$ as stated in Proposition 3.1, we obtain: if $u(t)=\bar{u}, \forall t \in(0, T)$, then

$$
x(t)=\left(x_{0}+\frac{b \bar{u}}{a}\right) e^{a t}-\frac{b \bar{u}}{a}
$$

if $u(t)=0, \forall t \in(0, T)$, then

$$
x(t)=x_{0} e^{a t}
$$

if $u(t)=0$ till $\tau$ and then $u(t)=\bar{u}$, we obtain

$$
x(t)= \begin{cases}x_{0} e^{a t}, & \text { if } t \in(0, \tau), \\ \left(x_{0}+\frac{b \bar{u}}{a} e^{-a \tau}\right) e^{a t}-\frac{b \bar{u}}{a}, & \text { if } t \in(\tau, T)\end{cases}
$$

finally, if $u(t)=\bar{u}$ till $\tau$ and then $u(t)=0$, the optimal state trajectory becomes

$$
x(t)= \begin{cases}\left(x_{0}+\frac{b \bar{u}}{a}\right) e^{a t}-\frac{b \bar{u}}{a}, & \text { if } t \in(0, \tau), \\ {\left[x_{0}+\frac{b \bar{u}}{a}\left(1-e^{-a \tau}\right)\right] e^{a t},} & \text { if } t \in(\tau, T) .\end{cases}
$$

Substituting (3)-(6) in the objective functional of problems $\left(\mathrm{P}_{\mathrm{q}}\right)$, taking into account Proposition 3.1, we obtain explicitly the function $F(q)$. 


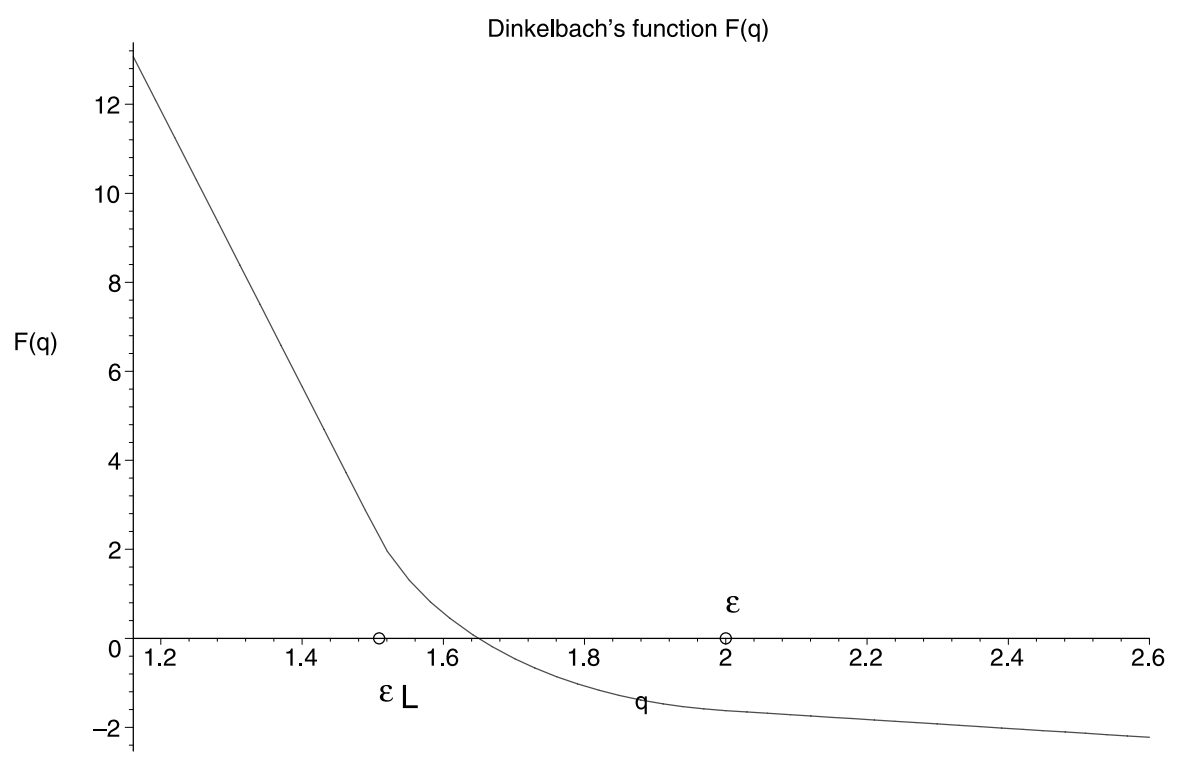

Fig. 1 Dinkelbach's function $F(q)$

We remark that the functions $F_{1}(q)$ and $F_{3}(q)$ are linear; this property will be used in the algorithm proposed below. With straightforward calculations, it is easy to prove that, if $b(k+a) \neq 0$, then the function $F(q)$ turns out to be not only continuous, which is true in the general case, but also differentiable.

As an example, let us consider the case $\bar{u}=30, k=3, c=0.10, T=1, a=-4$, $b=2, x_{0}=0.1, u_{0}=1$. Since $b(k+a)<0$, we have case (a) of Propositions 3.1 and 4.1. The function $F$ is

$$
F(q)= \begin{cases}-31 q+49.05677, & \text { if } q \leq 1.50916, \\ -8.5 q+15.37546+1.875(4 q-6) \log (2 q-3), & \text { if } 1.50916<q<2 \\ -q+0.37546, & \text { if } q \geq 2 .\end{cases}
$$

We plot the function $F(q)$ in Fig. 1. The unique zero of the function $F(q)$ lies in the interval $(1.50916,2)$; thus it can be found by solving the equation $F_{2}\left(q^{*}\right)=0$. The optimum value of problem $(\mathrm{P})$ is therefore the solution of the equation

$$
-8.5 q^{*}+15.37546+1.875\left(4 q^{*}-6\right) \log \left(2 q^{*}-3\right)=0 .
$$

A simple numerical computation allows us to find $q^{*} \simeq 1.64960$. From (2), we obtain the optimal switching time $\tau^{*} \simeq 0.69834$.

\subsection{Optimal Value of Problem (P)}

Remark that Proposition 3.1 allows us to determine the optimal control for problem (P) as soon as we know its optimum value $q^{*}$. In this section, we provide an algorithm to find $q^{*}$. 
While Dinkelbach's approach requires to solve the equation $F(q)=0$ where the function $F$ is usually known only implicitly, the special structure of problem $(\mathrm{P})$ allows to give the explicit expression of function $F$ for each value of $q$ (see Proposition 4.1). This sharply reduces the difficulty of the problem.

As a consequence of Proposition 4.1, using the monotonicity properties of Dinkelbach's function $F(q)$, it is possible to propose a procedure to find $q^{*}$ and consequently the optimal control $u^{*}$. The first step consists in computing the value of $b(k+a)$, which determines the analytical expression of function $F(q)$. Once $F$ is known, it is possible to understand to which interval its unique zero belongs by simple computation of the piecewise function $F$ at the endpoints of the disjoint intervals of its domain. The detailed procedure to solve $(\mathrm{P})$ is sketched in Fig. 2.

Referring to the procedure outlined in Fig. 2, we remark that the equations $F_{1}\left(q^{*}\right)=0$ and $F_{3}\left(q^{*}\right)=0$ are linear with respect to $q^{*}$, while to solve $F_{2}\left(q^{*}\right)=0$ and $F_{4}\left(q^{*}\right)=0$ it is possible to apply some well known numerical solution tech-

The optimal value $q^{*}$ and the optimal control $u^{*}$ of Problem $P$ can be found as follows:

if $b(k+a)<0$ then

if $F_{1}(b L) \leq 0$ then $F_{1}\left(q^{*}\right)=0$ and $u^{*}(t)=u \forall t \in(0, T)$

else if $F_{3}(b) \geq 0$ then $F_{3}\left(q^{*}\right)=0$ and $u^{*}(t)=0 \forall t \in(0, T)$

$$
\text { else } F_{2}\left(q^{*}\right)=0 \text { and } u^{*}(t)= \begin{cases}0, & \text { if } t \in\left(0, \tau^{*}\right) \\ u, & \text { if } t \in\left(\tau^{*}, T\right)\end{cases}
$$

if $b(k+a)>0$ then

$$
\begin{aligned}
& \text { if } F_{1}(b L) \leq 0 \text { then } F_{1}\left(q^{*}\right)=0 \text { and } u^{*}(t)=u \forall t \in(0, T) \\
& \qquad \begin{aligned}
\text { else if } F_{3}(b) \geq 0 \text { then } F_{3}\left(q^{*}\right)=0 \text { and } u^{*}(t)=0 \forall t \in(0, T) \\
\text { else } F_{4}\left(q^{*}\right)=0 \text { and } u^{*}(t)=\left\{\begin{array}{cc}
u, & \text { if } t \in\left(0, \tau^{*}\right) \\
0, & \text { if } t \in\left(\tau^{*}, T\right)
\end{array}\right.
\end{aligned}
\end{aligned}
$$

if $b(k+a)=0$ then

$$
\begin{aligned}
& \text { if } F_{1}(b)=F_{3}(b)=0 \quad \text { then } q^{*}=b \text { and any feasible control is optimal } \\
& \qquad \begin{array}{r}
\text { else if } F_{1}(b) \leq 0 \quad \text { then } F_{1}\left(q^{*}\right)=0 \text { and } u^{*}(t)=u \forall t \in(0, T) \\
\text { else } F_{3}\left(q^{*}\right)=0 \text { and } u^{*}(t)=0 \forall t \in(0, T)
\end{array}
\end{aligned}
$$

Fig. 2 An algorithm to solve problem (P) 
niques, e.g. a Newton-like method. In fact, the functions $F_{2}$ and $F_{4}$ are both decreasing, convex and $C^{\infty}$ in the intervals $(b L, b)$ and $(b, b L)$, respectively.

Recalling now the example presented in the preceding section, we observe that applying the algorithm it turns out that the optimal value $q^{*}$ can be found solving the equation $F_{2}(q)=0$, which confirms the result obtained simply looking at the numerical plot of the function $F$.

\section{Advertising Efficiency Maximization Problem}

In this section, we formulate the problem of maximizing the advertising efficiency of a firm during a selling period $[0, T]$, as a fractional control problem and use the results obtained in the previous sections in order to find the optimal advertising policies.

The problem of determining the optimal advertising policy has been largely analyzed in marketing literature by means of dynamic optimal control models (see $[9,10]$, for a review). The various advertising models essentially differ from each other in the dynamics which connects advertising to sales and in the objectives pursued by the firm.

We will consider the same dynamics as in the well known Nerlove-Arrow model in which advertising is considered as an investment that builds up an advertising stock (called goodwill by Nerlove and Arrow), this way taking into account the long term effects of advertising on consumers' demand (see [11]). But, unlike other advertising models, we focus on the concept of technical efficiency of the firm, defined as the ratio between the output produced by the firm and the input used in the production process (a sort of productivity ratio).

Let us denote the advertising stock at time $t$ by $\mathrm{A}(\mathrm{t})$ and the sales rate at time $t$ by $S(t)$. We assume as relevant outputs of the advertising investment, the final advertising stock $A(T)$ and the total sales during the selling period. As inputs to the firm's activity we consider total advertising costs and some fixed costs. Denoting by $a(t)$ the advertising expenditure rate at time $t$, we define the advertising efficiency as

$$
E=\frac{\alpha A(T)+(1-\alpha) \int_{0}^{T} S(t) d t}{C_{0}+\int_{0}^{T} a(t) d t},
$$

where $\alpha \in(0,1)$ represents the weight assigned to the final advertising stock and $C_{0}$ denotes fixed costs. Putting $k=(1-\alpha) / \alpha$, we can write the advertising efficiency as follows:

$$
E=\alpha \frac{A(T)+k \int_{0}^{T} S(t) d t}{C_{0}+\int_{0}^{T} a(t) d t},
$$

where the parameter $k>0$ is the weight assigned to the total sales.

We consider advertising stock (goodwill) dynamics as defined by

$$
\dot{A}(t)=-\delta A(t)+\epsilon a(t),
$$

as in the Nerlove-Arrow model [11], apart from the parameter $\epsilon>0$ that represents the advertising productivity in terms of the advertising stock. 
The sales rate $S(t)$ is defined as an affine transformation of the advertising stock at time $t$,

$$
S(t)=A(t)+b, \quad b \geq 0 .
$$

The advertising efficiency maximization problem is

$$
\begin{aligned}
\text { (EP) } \max & \frac{A(T)+k \int_{0}^{T}(A(t)+b) d t}{C_{0}+\int_{0}^{T} a(t) d t}, \\
\text { s.t. } & \dot{A}(t)=-\delta A(t)+\epsilon a(t), \\
& A(0)=A_{0}, \\
& a \in[0, \bar{a}],
\end{aligned}
$$

where $\bar{a}>0$, is the maximum advertising expenditure rate and $A_{0}$ is the initial advertising stock.

We can reread Proposition 3.1 in terms of optimal advertising policies. Indeed, in the EP problem we know the signs of parameters: $k>0, \epsilon>0, \delta>0, b \geq 0, \bar{a}>0$. Hence, from Proposition 3.1, we can distinguish two main cases.

If the decay rate of goodwill is high enough, thus meaning that the advertising forgetfulness is high enough (this situation corresponds to the case $\delta>k$ ), the optimal advertising policy $a(t)$ has the following structure:

$$
a(t)= \begin{cases}0, & \text { if } t \in(0, \tau), \\ \bar{a}, & \text { if } t \in(\tau, T),\end{cases}
$$

namely, it is convenient initially to start with no advertising, whereas it is convenient to undertake maximum advertising at the end of the communication period.

On the other hand, if $\delta<k$, that is the decay rate of the advertising stock is low enough, it is convenient to maximize the advertising effort from the very beginning and the optimal advertising policy $a(t)$ has in general the following form:

$$
a(t)= \begin{cases}\bar{a}, & \text { if } t \in(0, \tau), \\ 0, & \text { if } t \in(\tau, T) .\end{cases}
$$

Observe that the special structure of problem EP allows to obtain some properties of its optimum value function. In fact, the optimal value $q^{*}$ of EP is implicitly defined by the equation

$$
F\left(q^{*}\right)=0 .
$$

Following Sect. 4 it is easy to solve problem EP. Moreover, if $\delta \neq k$, function $F$ is differentiable and decreasing, therefore $\partial F / \partial q<0$ and it is possible to apply the implicit function theorem to obtain the sign of the derivative of $q^{*}$ with respect to the parameters. Straightforward calculations thus allow us to prove that:

$\frac{\partial q^{*}}{\partial b}>0, \quad \frac{\partial q^{*}}{\partial \delta}<0, \quad \frac{\partial q^{*}}{\partial A_{0}}>0, \quad \frac{\partial q^{*}}{\partial C_{0}}<0, \quad \frac{\partial q^{*}}{\partial \bar{a}} \geq 0, \quad \frac{\partial q^{*}}{\partial k}>0, \quad \frac{\partial q^{*}}{\partial \epsilon} \geq 0$. 


\section{Conclusions}

In this paper, we consider a fractional optimal control problem and solve it by means of Dinkelbach's approach. The particular structure of the functional allows to obtain an almost explicit solution of the problem. The same method can be used also in the case of more controls and/or more state variables. With one state variable and several controls all switching times can be explicitly obtained for each fixed $q$. In the case of several state variables switches can be computed but, in general, not in closed form.

The same methodology could be applied to more general classes of fractional functionals: this will be addressed in the next future research.

Moreover, the advertising problem presented in Sect. 5 could be generalized in order to compare different advertisers or different media in a Data Envelopment Analysis (DEA) framework. This could therefore lead to a dynamic approach to DEA, which will be another stimulating topic for future research.

\section{References}

1. Stancu-Minasian, I.M.: Fractional Programming Theory, Methods and Applications. Kluwer Academic, Dordrecht (1997)

2. Bhatt, S.K.: An existence theorem for a fractional control problem. J. Optim. Theory Appl. 11, 379385 (1973)

3. Miele, A.: On the minimization of the product of powers of several integrals. J. Optim. Theory Appl. 1, 70-82 (1967)

4. Dinkelbach, W.: On nonlinear fractional programming. Manag. Sci. 13, 492-498 (1967)

5. Pardalos, P.M., Phillips, A.T.: Global optimization of fractional programs. J. Glob. Optim. 1, 173-182 (1991)

6. Hager, W., Pardalos, P.M. (eds.): Optimal Control: Theory, Algorithms and Applications. Kluwer Academic, Dordrecht (1998)

7. Pardalos, P.M., Tseveendorj, I., Enkhbat, R. (eds.): Optimization and Optimal Control. World Scientific, Singapore (2003)

8. Sethi, S.P., Thompson, G.L.: Optimal Control Theory: Applications to Management Science and Economics. Kluwer Academic, Dordrecht (2000)

9. Sethi, S.P.: Dynamic optimal control models in advertising: a survey. SIAM Rev. 19, 685-725 (1977)

10. Feichtinger, G., Hartl, R., Sethi, S.: Dynamic optimal control models in advertising: recent developments. Manag. Sci. 40, 195-226 (1994)

11. Nerlove, M., Arrow, K.J.: Optimal advertising policy under dynamic conditions. Economica 29, 129142 (1962) 